American Journal of Infectious Diseases 6 (2): 40-43, 2010

ISSN 1553-6203

(C) 2010 Science Publications

\title{
Brain Abscess Complicating an Intracranial Hemorrhage
}

\author{
${ }^{1}$ M.D. Vikramjit Mukherjee and ${ }^{2}$ M.D. Maria Elena Ruiz \\ ${ }^{1}$ Department of Internal Medicine, \\ ${ }^{2}$ Department of Internal Medicine, Section of Infectious Diseases, \\ Washington Hospital Center, Washington, DC 20010, USA
}

\begin{abstract}
Problem statement: A brain abscess presenting after an Intracranial Hemorrhage (ICH) is an extremely rare occurrence. Diagnosis in this setting is difficult, given the absence of key clinical features, such as neurologic deficit and headaches. Since a delay in the diagnosis of a brain abscess leads to poor outcomes, there should be a high index of suspicion for abscess in patients developing fever after an ICH. Approach: This is a case report describing the presentation, hospital course and management of a patient who was diagnosed with a brain abscess following an ICH. Results: A review of literature revealed only 15 such cases. The basal ganglia are the most common sites of infection. The mean interval between the ICH and the systemic infection which resulted in an abscess was 9.8 days. Time from infection to brain abscess formation was 8-140 days, with a mean of 20 days. There was a delay in diagnosis in earlier case reports. Conclusion: Although brain abscess is a rare complication after ICH, because of the high mortality and morbidity, it should be considered in patients who develop fever after ICH.
\end{abstract}

Key words: ICH, abscess, Serratia, CVA

\section{INTRODUCTION}

Brain abscess complicating an ICH is extremely rare, with only fifteen such cases extracted from a Medline search. A brain abscess is a neurological emergency, requiring prompt administration of high dose parenteral antibiotics and surgical evaluation. Most of the patients reported earlier had an identifiable source of infection with transient bacteremia, following which the patient's neurological status started to decline (Nakai et al., 2006). This suggests that brain abscess complicating an ICH is due to hematogenous seeding of a region made vulnerable due to destruction of the blood brain barrier.

Case report: A 68 year old woman with well controlled hypertension and diabetes presented to an outside hospital with new onset left sided weakness. An MRI showed acute right basal ganglia infarct with no signs of hemorrhage. Around $24 \mathrm{~h}$ after the initial onset of symptoms, the patient was noted to have worsening neurological status and a repeat Computed Tomography (CT) scan revealed a right basal ganglia hematoma with intraventricular extension and midline shift. She was intubated for airway protection and urgently transferred to the Washington Hospital Center. On initial examination, the patient was noted to be sedated and intubated, with complete left hemiparesis. An External Ventricular Drain (EVD) was placed on the contralateral side. She was extubated successfully 1 week after admission and received an eight day course of vancomycin and piperacillin-tazobactam for aspiration pneumonia in the intensive care unit. The EVD drained clear serosanguineous fluid and was removed ten days after admission. The patient's neurological status was stable during this interval, as were the CT images of her ICH (Fig. 1A). Ten days after admission, the patient developed fever of $101.8^{\circ} \mathrm{F}$ and was noted to have respiratory failure and worsening neurological status. The patient was reintubated and respiratory cultures grew Serratia marcescens. Fever subsided four days post initiation of antibiotic regimen for health care associated pneumonia, accompanied by gradual improvement of mental status. CT findings during this time revealed resolving hematoma with decrease in the surrounding edema (Fig. 1B).

On hospital day 30, the patient developed high grade fevers again. She was noted to have a temperature of $103.4^{\circ} \mathrm{F}$ and this was again accompanied by worsening neurological status. Contrast-enhanced CT of her head showed interval increase in the size of the hematoma with ring enhancement and edema around the ICH (Fig. 1C).

Corresponding Author: M.D. Vikramjit Mukherjee, Department of Internal Medicine, Washington Hospital Center, Washington, DC 20010, USA 


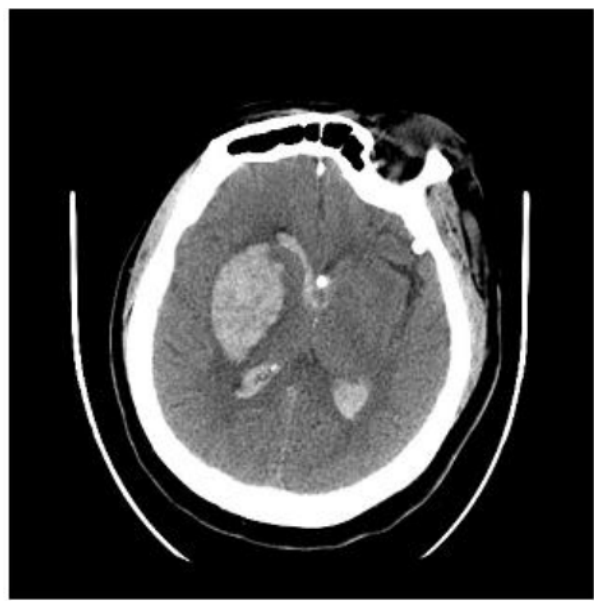

Fig. 1A: Initial presentation of the patient following hemorrhagic transformation of a right ganglia infarct

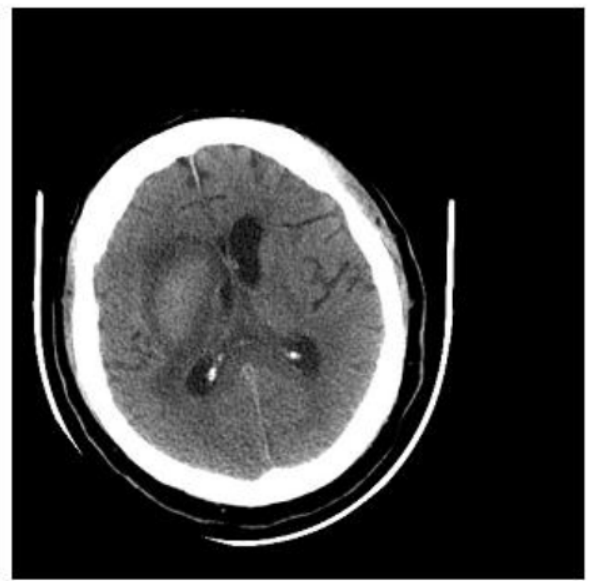

Fig. 1B: Gradual resolution of the hemorrhage

Given the possibility of a brain abscess, a stereotactic biopsy of her hematoma was performed. Around $10 \mathrm{~mL}$ of old blood was removed and acute on chronic inflammation with clumps of bacteria and hemosiderin was noted by Pathology. The specimen grew Serratia marcescens on culture, with a sensitivity pattern similar to the earlier respiratory isolate. The patient was started on Ceftriaxone since the isolate was sensitive to it and due to its excellent central nervous system penetration. Three days later, the patient had a downward trend of her white cell count, resolution of her fevers and an improvement in neurological status. CT scans done 2 and 4 weeks after the biopsy revealed marked decrease in the size of the right basal ganglia abscess and resolution of the vasogenic edema surrounding the lesion (Fig. 1D).

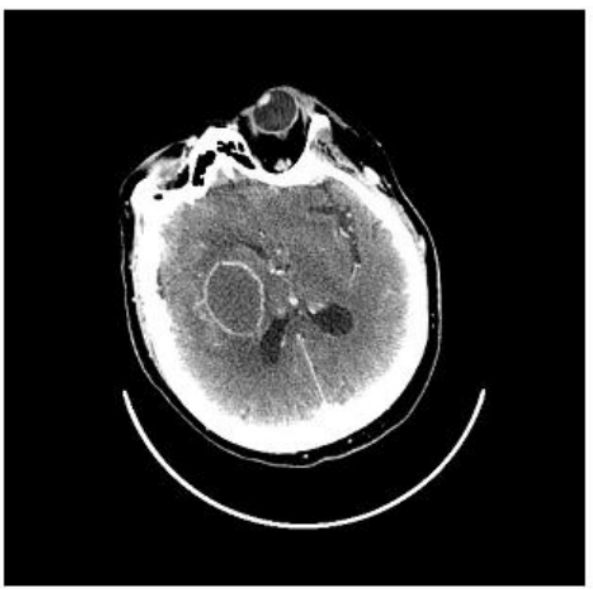

Fig. 1C: Development of a ring enhancing lesion at the site of the ICH

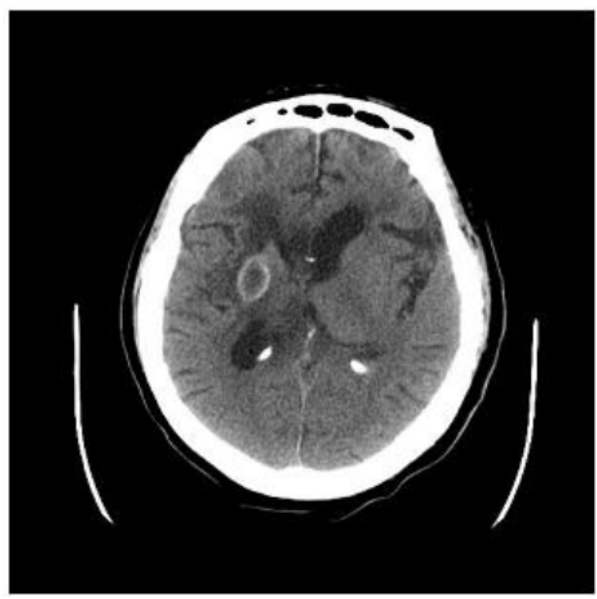

Fig. 1D: Resolution of the abscess following appropriate antibiotics

\section{MATERIALS AND METHODS}

Data was gathered from the chart once IRB approval was obtained. A Pubmed review revealed to us previously published reports describing similar cases.

\section{RESULTS}

Computed Tomography images revealed marked changes during the patient's hospital stay. As described in the case report, the patient's neurological status deteriorated initially during the episode of pneumonia and then again during the development of the brain abscess. 


\section{DISCUSSION}

Brain abscesses have a mortality rate of $15 \%$ and require urgent surgical evaluation (Goldman et al., 2007). Factors associated with a poor prognosis include extremes of age and diagnostic delay. While the classical clinical features have been described as fever, headache and focal neurological deficits, this triad may be present in less than $20 \%$ of the cases (Tseng and Tseng, 2006). Brain abscess following an intracranial hemorrhage is even more difficult to diagnose clinically unless a high index of suspicion is maintained.

Brain abscess following an intracranial hemorrhage is an extremely rare phenomenon with only fifteen such cases reported in English literature (Nakai et al., 2006). Of note, most of these cases have been reported from Asia and Europe and this is the first one to be reported from North America. Disruption of the blood-brain barrier caused by hemorrhage may predispose the brain tissue to infection and subsequent abscess formation (Chen et al., 1995). Host factors such as diabetes mellitus and renal failure have also been shown to predispose a patient to this complication (Arentoft et al., 1993; Davenport et al., 1995). Since the ganglia are nested away from the usual sources of infection, ganglionic abscesses are thought to be hematogenous in origin (Molavi and Dinubile, 1988). A literature review suggested that the basal ganglia are the most common site of an abscess complicating an ICH. The source of infective foci is usually a distant site, with the thorax being the most common location (Molavi and Dinubile, 1988), with Staphylococcus being the most common organism (Nakai et al., 2006).

In our case, Serratia spp. was isolated from both the abscess and from respiratory cultures during the patient's pneumonia. Hence we hypothesize that hematogenous spread from the pneumonia led to bacterial seeding of the $\mathrm{ICH}$, leading to the formation of the brain abscess, in the setting of impaired host defense mechanisms. In previously reported cases, the mean interval between the ICH and the systemic infection which ultimately resulted in a brain abscess was 9.8 days (Nakai et al., 2006); however it could be as delayed as 55 days (Molavi and Dinubile, 1988). In our case, the pneumonia developed 10 days after the ICH. Our patient had multiple risk factors for the development of an infection, including old age, diabetes, renal failure, emergent intubation and central venous line insertion. In our case, the time between the systemic infection and subsequent neurological deterioration suggestive of a brain abscess was 20 days, while earlier reports showed a range of 8-140 days (Nakai et al., 2006; Nowak and Rodiek, 2003).
There was a delay of 3 weeks between the probable date of abscess development and confirmatory diagnosis via stereotactic biopsy. This was because of the rarity of the condition, an effort to rule out more common causes of fever in a hospitalized patient and the absence of overt clinical features of headache and new focal neurological deficit. There has been a delay in diagnosis in the earlier case reports as well (Lee et al., 1994), thus emphasizing that a high index of suspicion be maintained whenever ICH patients with a recent history of systemic infection develop progressive fever and deteriorating levels of consciousness (Beloosesky et al., 2002). The treatment of choice for these patients remains aspiration and medical therapy; however some cases have responded to medical therapy alone (Amayo et al., 2002).

\section{CONCLUSION}

While brain abscess after ICH is extremely rare, it is an important diagnosis to consider when patients with basal ganglia hemorrhages develop fever. There is usually a delay in diagnosis and an extra cranial source, usually the lungs, can usually be identified. Prompt identification of this complication should result in earlier appropriate management and lower mortality and morbidity.

\section{REFERENCES}

Amayo, E.O., T.O. Kwasa, C.K. Musau, N. Mugo and J. Wambani, 2002. Primary intracerebral haemorrhage complicated by cerebral abscess: Case report. East Afr. Med. J., 79: 163-164. PMID: 12389964

Arentoft, H., H. Schonheyder and N.K. Schonemann, 1993. Cerebral salmonella typhimurium abscess in a patient with a stroke. Infection, 21: 251-253. PMID: 8225631

Beloosesky, Y., J.Y. Streifler, N. Eynan and J. Grinblat, 2002. Brain abscess complicating cerebral infarct. Age Age., 31: 477-480. PMID: 12446296

Chen, S.T., L.M. Tang and L.S. Ro, 1995. Brain abscess as a complication of stroke. Stroke, 26: 696-698. PMID: 7709419

Davenport, R.J., R. Gibson, C.J. Mumford and R.G. Will, 1995. Brain abscess complicating ischemic stroke. Stroke, 26: 1501-1503. PMID: 7631361

Goldman, L., D.A. Ausiello, W. Arend, J.O. Armitage and D. Clemmons et al., 2007. Goldman Cecil Medicine Expert Consult. 23rd Edn., Saunders, US., SBN: 13: 978-1416028055, pp: 3120. 
Lee, K.S., W.K. Bae, H.G. Bae J.W. Doh and I.G. Yun, 1994. Brain abscess from a ganglionic hemorrhagea case report. J. Korean Med. Sci., 9: 259-263. PMID: 7993595

Molavi, A. and M. Dinubile, 1988. Brain Abscess. In: Handbook of Clinical Neurology, Vinken, P.J., G.W. Bruyn and H.L. Klawans (Eds.). North Holland Publishing Company, Amsterdam, ISBN: 13: 978-0-444-50917-8, pp: 143-166.

Nakai, K., T. Yamamoto, S. Yasuda and A. Matsumura, 2006. Brain abscess following intracerebral haemorrhage. J. Clin. Neurosci., 13: 1047-1051. PMID: 17664069
Nowak, D.A. and S.O. Rodiek, 2003. Topka Pyogenic brain abscess following haematogenous seeding of a thalamic haemorrhage. Neuroradiology, 45: 157-159. PMID: 12684718

Tseng, J.H. and M.Y. Tseng, 2006. Brain abscess in 142 patients: Factors influencing outcome and mortality. Surg. Neurol., 65: 557-562. PMID: 16720170 